\title{
Road surface mirage: A bunch of hot air?
}

\author{
ZHOU HuaiChun $^{1 *}$, HUANG ZhiFeng ${ }^{1,2}$, CHENG Qiang $^{1}$, LÜ Wei $^{1}$, QIU Kui ${ }^{1}$, CHEN Chen $^{1} \&$ \\ HSU Pei-feng ${ }^{2}$ \\ ${ }^{1}$ State Key Laboratory of Coal Combustion, School of Energy and Power Engineering, Huazhong University of Science and Technology, Wuhan \\ 430074, China; \\ ${ }^{2}$ Department of Mechanical and Aerospace Engineering, Florida Institute of Technology, Melbourne, FL 32901-6975, USA
}

Received September 25, 2010; accepted November 14, 2010

\begin{abstract}
The inferior mirage from road surfaces is a common phenomenon, which can be easily seen in everyday life. It has been recognized in the literature as a light refraction phenomenon due to the refractive index gradient caused by the temperature gradient in the air strata above the road surfaces. However, it was also suggested that the mirage is just a phenomenon of specular reflection at grazing incidence. Because of the lack of reasonable and quantitative evidence, the generally accepted light refraction theory has not yet been refuted. Here we show some mirror-like reflection images captured from a road surface stretch in Yujiashan North Road, Wuhan, China, when there was no obvious temperature gradient on or above the road, measured on a winter day in December 2009. This provided direct evidence to doubt the temperature induced light refraction mechanism of the inferior mirage. Furthermore, the critical grazing angle of about $0.2^{\circ}$ to the road plane where the mirror-like reflection appears could not make the rough surface scatter incident light as a smooth surface according to the Rayleigh criterion. Therefore the phenomenon is a mirrorlike observation effect of scattering from the surface, which cannot be entirely explained by light refraction via air strata. The results demonstrate that the image-formation mechanism and the observer-based-analysis method shown here potentially offer a means of understanding a wide range of scattering phenomena from rough surfaces at grazing angle; for example, the superior mirages of unusual brightness occasionally observed over frozen lakes and the off-specular reflection phenomenon.
\end{abstract}

inferior mirage, rough road surface, light reflection, light refraction, Rayleigh criterion

Citation: Zhou H C, Huang Z F, Cheng Q, et al. Road surface mirage: A bunch of hot air? Chinese Sci Bull, 2011, 56: 962-968, doi: 10.1007/s11434-011-4347-9

Have you heard of mirages occurring over the surface of the sea or a desert? Have you noticed mirages appearing on the highway at a distance far away from you? The answer to these questions for most of us is yes. The mirages from the surface of a highway, pavement or sidewalk have been frequently reported [1-3] and were first discussed in the journal Science around 1920 [4-6]. The mirages were generally attributed to the graded refractive index in the air strata with a graded temperature distribution over a hot road surface, and to this date, this concept has been widely accepted in the literature [1-13]. Some computer simulation work has also been published attempting to reconstruct the phenomena with seemingly successful results [14].

*Corresponding author (email: hczhou@mail.hust.edu.cn)
However, doubt has also been cast on the inferior mirage explanation of the phenomena for a similarly long period of time [15-18]. Platt [15] stated that the sidewalk mirage is a phenomenon of simple reflection, being entirely independent of atmospheric conditions. Riggs [16] thought that the water-like phenomenon on the highway together with reflections of cars and roadside objects was probably because of regular reflection by the road surface at grazing incidence. Tavassoly et al. [18] reported that they had measured the temperature variations in the air layers adjacent to the road surface and the distance from the mirage to the observer for several such mirages that could be observed in the area, and they found that the requirements for total internal reflection were not satisfied. However, partially because of the lack of reasonable and quantitative evidence, the gener- 
ally accepted light refraction theory has not yet been totally refuted.

The propagation of electromagnetic waves inside media and their interaction with complicated surfaces has been studied by many researchers [19-21]. For the scattering of electromagnetic waves from rough surfaces, it is common knowledged that if the incident angle and the scattering angle are large, approaching the grazing angle, specular reflection would occur according to the Rayleigh criterion [22-25]. However, it has not been examined whether the mirror-like reflection from a rough road surface is specular reflection phenomenon obeying the Rayleigh criterion. The interests in random roughness surfaces are quite diverse. For example, we have conducted extensive work on ramifications of microscale roughness surfaces in thermal radiative environment [26-28]. The present study is devoted to investigating the mechanism of formation of a road surface mirage with directly measured evidence.

\section{Observation results and analysis}

\subsection{The observation conditions and results}

Some typical mirror-like reflection images on summer and winter days, shown in Figure 1(a) and (c), respectively, were captured from a stretch on Yujiashan North Road (profile in Figure 1(e)), a local road at the foot of Yujiashan Hill in Wuhan, China. The road stretch consists of a descending part, with a slope of about -0.02 , from the observation point I, where the images were taken, and an ascending part ending at point II. The camera used was a Canon EOS 500D. The image capturing parameters for Figure 1(a) were: Date/time: 2009-07-12 11:26:15; Exposure time: 1/400 s; F-number: 8; ISO: 100; Focal length: $187 \mathrm{~mm}$. The image capturing parameters for Figure 1(c) were: Date/time: 2009-12-19 12:08:57; Exposure time: 1/250 s; F-number: 16; ISO: 400; Focal length: $250 \mathrm{~mm}$. The temperature gradients over the road surface in the summer day (Figure 1(b)) and in the winter day (Figure 1(d)) were measured as the images in Figure 1(a) and Figure 1(c) were captured. The measurement conditions for Figure 1(b) were: Date: 2009-07-12; Time: 13:12-13:41; Weather: Sunny; High temperature: $35^{\circ} \mathrm{C}$; Low temperature: $28^{\circ} \mathrm{C}$; Wind: $\mathrm{SE}$ $2.0-5.0 \mathrm{~m} / \mathrm{s}$. The measurement conditions for Figure 1(c) were: Date: 2009-12-19; Time: 10:50-11:15; Weather: Sunny; High temperature: $7^{\circ} \mathrm{C}$; Low temperature: $0^{\circ} \mathrm{C}$; Wind: NW $0.3-3.0 \mathrm{~m} / \mathrm{s}$. The observation point I was offset $0.6 \mathrm{~m}$ from the white and solid line on the south-side to the road center, and its height is $0.65 \mathrm{~m}$ above the road surface.

From the images in Figure 1(a) and (c) it is clear that the double-yellow and solid lines in the center of the road are truncated and reflected approximately specularly. At the same time, the cars passing through the area with reflected lines also show clear reflected images under the road surface. Even in summer evenings, we also easily observe the reflected double-yellow and solid lines.

Now we examine the influence of the hot air above the road on the mirror-like reflection. The temperature profiles on and above the road surface displayed in Figure 1(b) and (d) were measured using a copper-constantan thermocouple with an uncertainty less than $1^{\circ} \mathrm{C}$. The mean temperature at every elevation in the figure was obtained from 50 or more measurements, which were taken at a $2-\mathrm{Hz}$ sampling rate and the maximum standard deviation is less than $1{ }^{\circ} \mathrm{C}$. From Figure 1(b), it can be seen that the temperature on the road in the summer day is about $7^{\circ} \mathrm{C}$ higher than that in the air 10 $\mathrm{mm}$ over the surface, and the air temperature does not change significantly at higher elevations. A similar observation was reported by Ramadas and Malurkar [29].

However, we found that there was no obvious temperature difference on and over the road surface in the winter day (Figure 1(d)). Nevertheless, we captured clear mirrorlike reflection images on that day (Figure 1(c)). This clearly contradicts the commonly accepted mechanism of the inferior mirage phenomenon caused by the temperature gradient above a road surface.

From our observations it is also seen that the mirror-like reflection is not disturbed by the passing of cars. The mirror-like reflection is a common phenomenon which can be easily seen everywhere from a grazing angle. We have captured many reflection images around the campus of Huazhong University of Science and Technology within three kilometers of point I. Holding the camera 5 to $15 \mathrm{~cm}$ over the ground, similar reflected images could be captured from the road surfaces 10 to $20 \mathrm{~m}$ ahead.

It is not the case that we can observe the mirror-like reflection at any time under any condition for the same road stretch where the reflection appeared. We failed to capture the images from the road in Figure 1(e) before 12 a.m. on a winter morning. At that time the sun is hanging in front of us, and the sun light scattered by the road surface is stronger than that from the cars and the road lines. We also did not capture the mirror-like reflection from the same place after about 4 p.m. on a winter day. At that time, the road surface is shadowed by Yujiashan Hill to the south. In the scattered light by the road surface, the part from of the cars and road lines was weaker than that directly from the background sky. As shown in Figure 2(a), the mirror-like reflection is a kind of secondary scattering phenomenon of the light from the background, and it is easily covered by the primary scattering light from the road surface under certain conditions. Therefore the mirror-like reflection can only be observed on a road stretch under the right environmental light conditions.

It can now be concluded that the inferior mirage seen from a rough road surface is a specular reflection from the surface at a grazing angle, not caused by the refractive index gradient in the air strata over the road. The latter, at best, is a secondary effect. 


\subsection{Analysis of the phenomenon}

The scattering of electromagnetic waves from rough surfaces at large incident and scattering angles is especially complicated [22-25]. We can check if the scattering angles in Figure 1(a) and (c) satisfy the Rayleigh criterion, which can be used to determine if a given reflection is specular using the factor $g$ [25]:

$$
g=\frac{4 \pi \sigma_{h} \cos \left(\theta_{i}\right)}{\lambda}
$$

where $\sigma_{h}$ is the roughness of the surface, $\lambda$ the wavelength, and $\theta_{i}$ the incident angle. If $g<<1$, then reflection is likely to be specular, and if $g>>1$, then, the reflection is diffuse.

The grazing angle $\alpha$ of the observation point I, shown in Figure 2(a), was determined from the local road stretch where a mirror-like reflection occurred in Figure 1(a) and (c). For this purpose, we used a pole standing on the road to mark the far point IV (Figure 2(b)), and the near point III (Figure 2(c)), of the reflected images, and the distances from points I to IV and III are 275 and $92 \mathrm{~m}$, respectively. The calculated results for the grazing angle $\alpha$ are shown in Figure 2(d). It can be seen that the grazing angles for the observer in the point I to the points III and IV are nearly equal $\left(0.2^{\circ}\right)$. Then $\theta_{i}=(90-0.2)^{\circ}=89.8^{\circ}$.

The roughness of the road surface was measured using a digital level instrument on July 13 and 14, 2009. The measurement was made on the road surface at a distance of 280 $\mathrm{m}$ from point $\mathrm{I}$, close to point IV. Four surface squares with dimensions of $10 \mathrm{~cm} \times 10 \mathrm{~cm}$ were measured with $5-\mathrm{mm}$ increments on each axis, and the total measurement points for each square are $21 \times 21=441$. The height measurement uncertainty was less than $0.1 \mathrm{~mm}$. For the road surface, $\sigma_{h}$ varied from $0.5 \mathrm{~mm}$ to $1.0 \mathrm{~mm}$ with a mean value of 0.7015
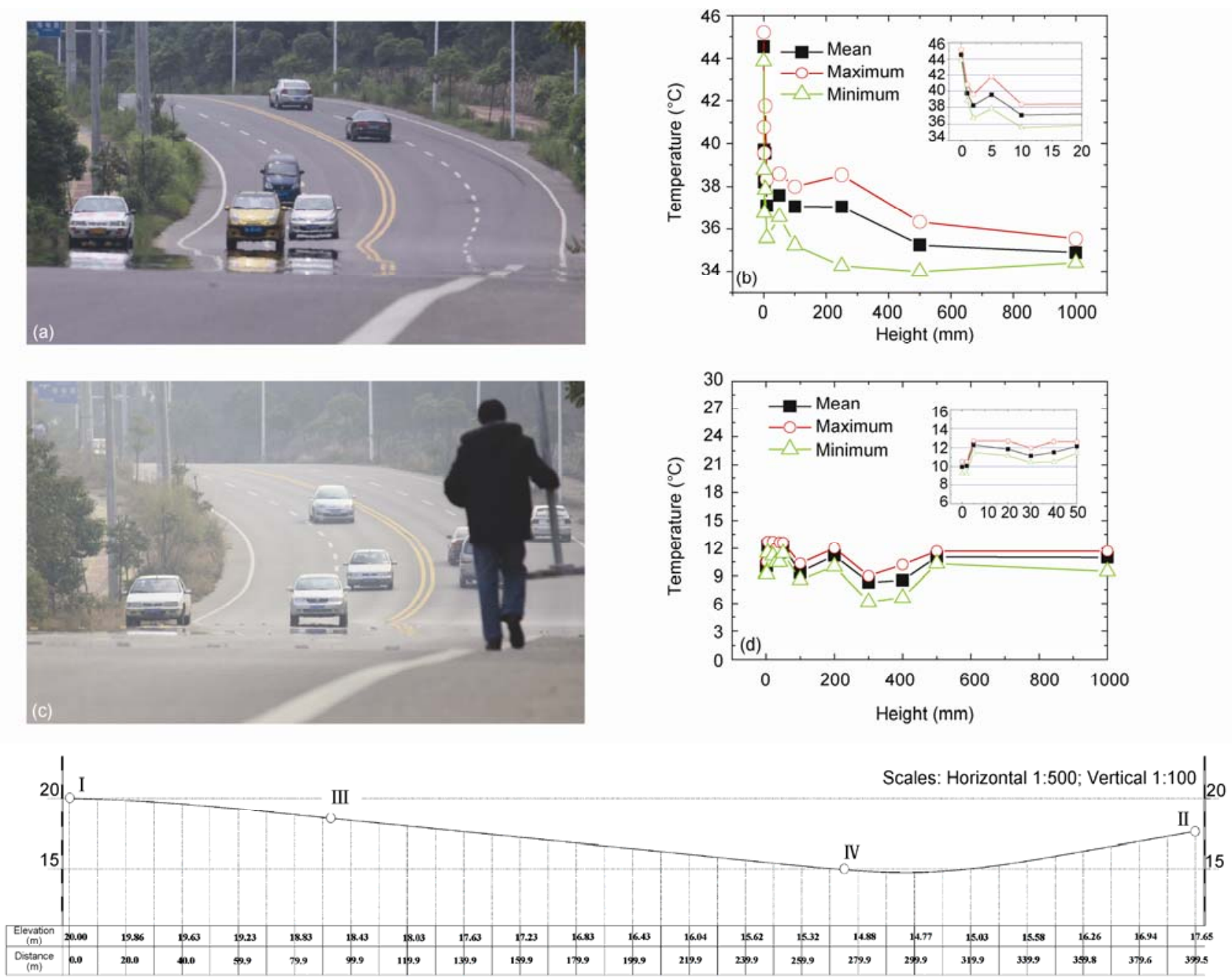

(e)

Figure 1 Mirror-like reflection images on a summer day (a) and on a winter day (c). The temperature gradients over the road surface on the summer day (b) and on the winter day (d) were measured as the images in (a) and (c) were captured. (e) The profile of the road stretch under observation, where point I (longitude: $114^{\circ} 25^{\prime} 01^{\prime \prime}$, latitude: $30^{\circ} 31^{\prime} 33^{\prime \prime}$ ) is the observation point, and point II is the most distant visible point on the road. The points III and IV are the beginning and end points of the specular reflection on the road surface, which are shown in Figure 2(c) and (b), respectively. 

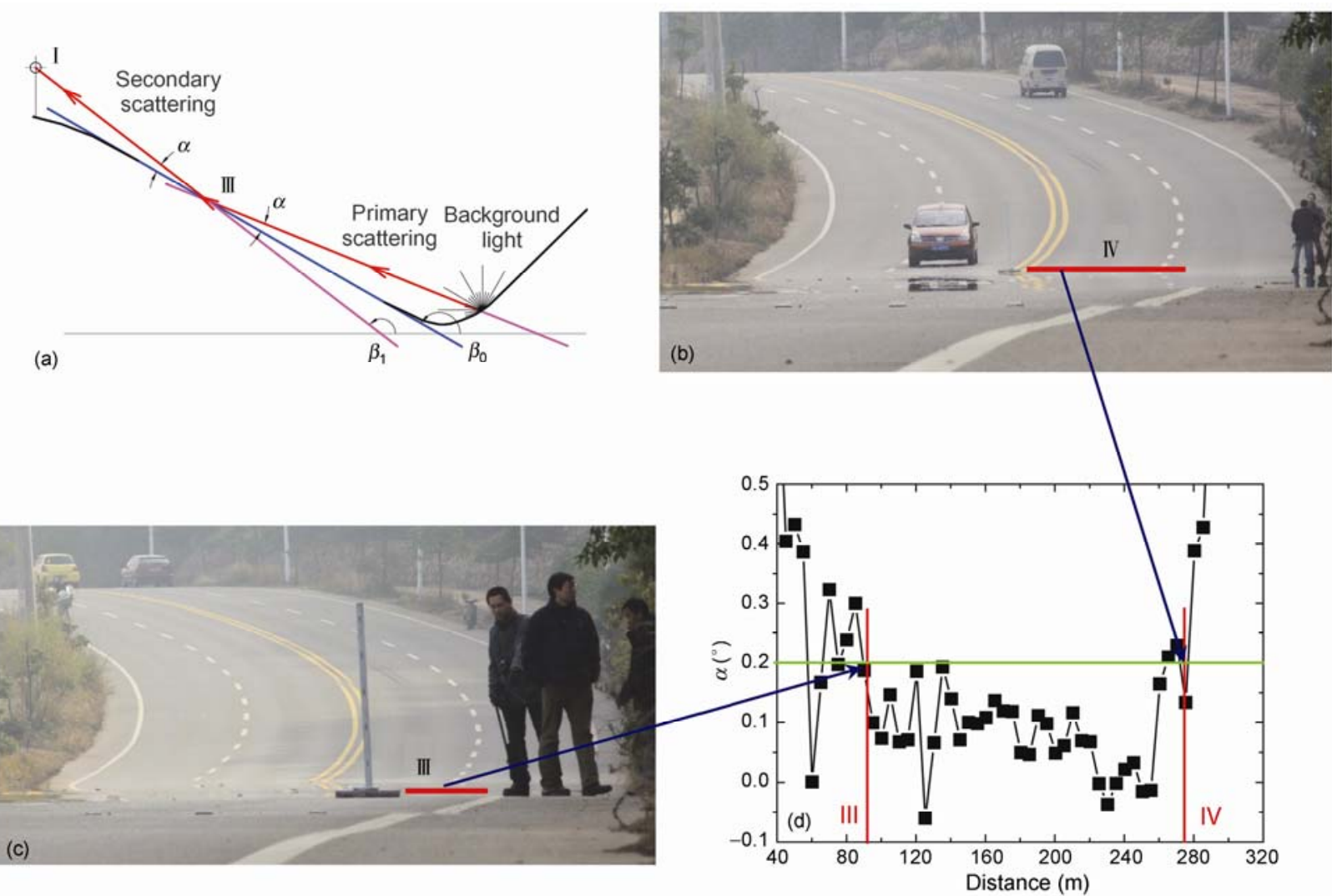

Figure 2 (a) The grazing angle for the observation point I from the local road plane. $\alpha=\beta_{0}-\beta_{1}$, where $\beta_{1}$ and $\beta_{0}$ are the angles of the observation line from point I to point III (or IV, or any point between III and IV), and the tangent plane at point III, respectively. This shows that the mirage observed at point I is just the secondary scattering light from the background light. (b) The far point IV, $275 \mathrm{~m}$ away from point I, and (c) point III, $92 \mathrm{~m}$ away, are the boundaries of the span of the specular reflection on the road surface. (d) The grazing angle varies with the distance from the observation point I. The deviation angles for points III and IV are nearly equal at $0.2^{\circ}$.

$\mathrm{mm}$ and $\sigma_{l}$, the mean distance between adjacent peaks in the profile, was about $1.72 \mathrm{~cm}$. Therefore, $\sigma_{l} / \sigma_{h} \approx 24.5$. For the visible wavelength of $400-800 \mathrm{~nm}$, the factor $g$ for the rough road surface is

$$
27.42<g<98.70 .
$$

From this result, it can be seen that even though the surface is not perfectly diffuse, it is far from specular. It is easy to estimate for the critical grazing angle which guarantees the road surface acting as a specular surface under the Rayleigh criterion. It turns out that the angle is less than $0.01^{\circ}$. This is significantly smaller than $0.2^{\circ}$, the critical angle by which the mirror-like reflection has been observed from the surface. Obviously, the phenomenon studied in the present study only appears in the far-field circumstance, and it depends on the observation conditions.

\section{Road mirage formation models}

We employ the validation conditions of the geometricoptics (GO) ray-tracing method to analyze the interaction between incident light waves and rough surfaces discussed in $[30,31]$. From the above analysis, parameters used in the regime map are: $\sigma_{h} / \sigma_{l}$ is about 0.04 , and $\sigma \cos \left(\theta_{i}\right) / \lambda$ is about 3.06 to 6.12 in visible light area [31]. The GO ray-tracing method is valid and accurate for the analysis of the light scattering from rough surfaces that are considered in this case. In a similar way, image processing techniques are effective for the characterization of scattering/reflecting in the radiative transfer process [32]. Based on the GO ray-tracing concept and the image formation process, a mechanism to explain the mirror-like reflection from a rough road surface is proposed in Figure 3. On a rough surface with a relatively low slope, or $\sigma_{l} / \sigma_{h}>1$, the local area around a peak is relatively smooth, which is true especially for the road surface. As shown in Figure 3(a) without any temperature gradient, the specular component of the reflection from the peaks can form a "continuous" mirror-like reflection image seen by an observer at a certain distance away under the following conditions:

(1) The specular component of an object scattered from at least three or more peaks can arrive simultaneously at the eyes of an observer;

(2) The distance between the reflection surface and the observer is far enough so that the discontinuous specular 

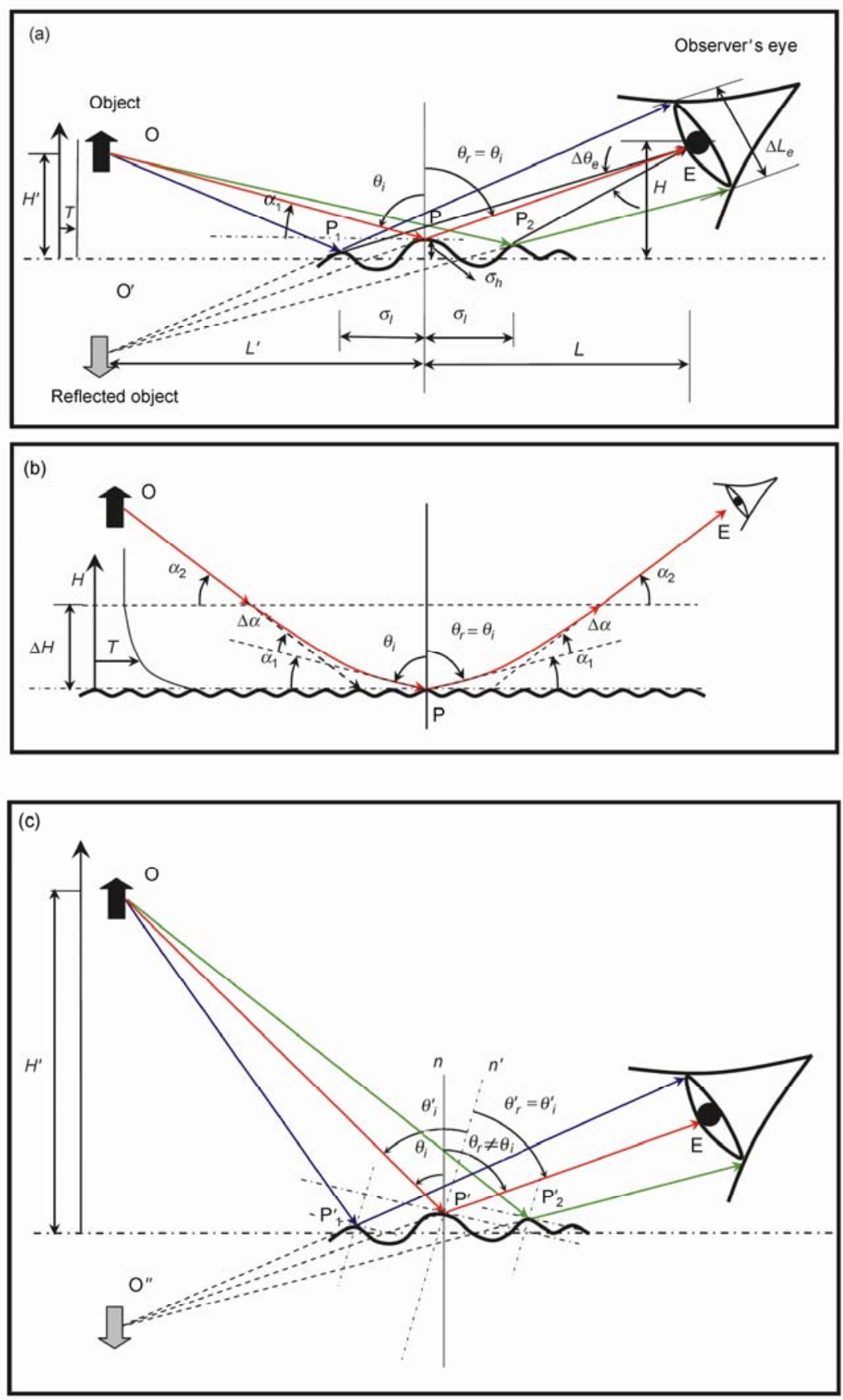

Figure 3 Mechanism of mirror-like reflection from rough road surfaces. (a) Without a temperature gradient; (b) with a temperature gradient. In (a), the scattered light from the adjacent peaks $\mathrm{P}_{1}, \mathrm{P}$ and $\mathrm{P}_{2}$ arrives at the eye simultaneously. $\Delta \theta_{e}$ is the incline angle between the sight lines, $\mathrm{P}_{1}$ and $\mathrm{P}_{2}$, to the eye. $\theta_{i}$ $=\theta_{r}=90-\alpha_{1}$ is the minimum incident/scattering angles from the rough surface normal, and $\alpha_{1}$ is the grazing angle. In (b), the deviation angle $\Delta \alpha$ caused by the refractive effect of the air layer with temperature gradient increases the total grazing angle to $\alpha_{2}=\alpha_{1}+\Delta \alpha$. (c) Light-enhancing effect at a grazing angle. When the reflection angle to the normal $n, \theta_{r}\left(\theta_{r} \neq \theta_{i}\right.$ and $\left.\theta_{r}>\theta_{i}\right)$, is larger than $80^{\circ}$, there exists a point $\mathrm{P}^{\prime}$ on the surface with a normal $n^{\prime}$ that makes a specular reflection of the light from $\mathrm{O}$ for the observer at E. $\theta_{r}{ }^{\prime}=\theta_{i}{ }^{\prime}$ and $\theta_{r}{ }^{\prime}+\theta_{i}{ }^{\prime}=\theta_{r}+\theta_{i}$. Larger reflecting angles ensure that the received reflectance from many points on the surface are within the resolution limits of a normal observer.

reflection from the adjacent peaks cannot be distinguished by the eye of the observer;

(3) The forward scattering dominates the scattering from the surface at large incident/scattering angles;
(4) The light of the background sky or the sun directly scattered from the surface at the grazing angle is weaker than the secondary scattering light from the surface at the same grazing angle which comes from the primary scatter- 
ing light from the object.

If a stable air layer with a temperature gradient exists over the road surface, as shown in Figure 3(b), the refractive effect of the air layer will cause an additional deviation angle $\Delta \alpha$, which makes the total deviation angle for light reflected from the road surface increase to $\alpha_{2}=\alpha_{1}+\Delta \alpha$. In that case, an observer would be able to see a wider area of "reflecting surface." This case may occur from a hot desert surface in summer without winds, where no moving objects on the surface will disturb the stable air layer. However as we have observed over the road surface, the heavy traffic breaks the air layer over it and any stable air layer with temperature gradient would not exist for some time, as was previously argued by Riggs [16].

Furthermore, the image-formation mechanism and the observer-based-analysis method shown here potentially offers a means of understanding a wide range of scattering phenomena from rough surfaces at grazing angles. For example, the superior mirages of unusual brightness occasionally observed over a frozen lake were reported [33]. In this case, at grazing angle of $0.046^{\circ}$, the luminance of the image is stronger than that of the diffuse scattering from the surface. Another example is the off-specular reflection phenomenon for both a metal and a non-metal rough surface [34]. In these cases, the enhanced reflectance at the grazing angles can be explained by the mechanism shown in Figure 3(c). Consider the reflectance angle to the normal $n, \theta_{r}\left(\theta_{r}\right.$ $\neq \theta_{i}$ and $\theta_{r}>\theta_{i}$ ), larger than $80^{\circ}$ and/or even approaching $90^{\circ}$. There exists a point $\mathrm{P}^{\prime}$ on the surface with a normal $n^{\prime}$ that makes a specular reflection for the light from $\mathrm{O}$ to the observer at E. As $\theta_{r}$ approaches $90^{\circ}$, there are an increasing number of points like $\mathrm{P}^{\prime}$ on the adjacent peaks within the resolution limit of a human's eyes, which results in a lightenhancing effect for the off-specular reflection at large reflectance angles. For the observation of the bright superior mirage [33], there is no special object in the sky background which can be seen by the observer, and the reflected lights in the bright mirage come from the sky with different incident directions. As shown in Figure 3(c), the reflectance of the lights by the lake ice will aggregate in the scattering angles larger than $89^{\circ}$, resulting in the bright mirage. The phenomenon is also not dominated by the refractive index gradient in the air over the lake ice.

\section{Conclusion}

The mechanisms of reflectance at grazing angles can be summarized as below. The phenomenon of image formation, which becomes a mirage seen from rough road surfaces and in deserts, comes from light scattered from an object with an incident angle equal to the reflectance angle. This is a specular reflection to the normal of the road surface plane. In this case, the grazing angle is normally less than $1^{\circ}$. If the incident light comes from an incident angle much smaller than $90^{\circ}$, for example, $60^{\circ}$, the reflectance becomes stronger as the scattering angle approaches $90^{\circ}$. This is caused by the light-enhancing effect, which results in the off-specular reflection [34]. The bright superior mirage observed from the lake ice [33] may be another kind of off-specular phenomena. However, these inferences will need further research and quantitative validation in the future.

This work was supported by the National Natural Science Foundation of China (50636010, 50721005) and the Program of Introducing Talents of Discipline to Universities of China (B06019).

1 Gove P B. Webster's Third New International Dictionary of the English Language Unabridged. Springfield, Massacusetts: G. \& C. Merriam Company, 1964. 1441

2 Lemons D S. Perfect Form: Variational Principles, Methods, and Applications in Elementary Physics. New Jersey: Princeton University Press, 1997. 33-37

3 Adam J A. A Mathematical Nature Walk. New Jersey: Princeton University Press, 2009. 73-77

4 Knowton A A. An unusual mirage. Science, 1919, 50: 328

5 McNair F W. A sidewalk mirage. Science, 1920, 52: 201

6 Du Shane C P. The sidewalk mirage. Science, 1921, 53: 236

7 Odell A F. Sidewalk mirages. Science, 1921, 54: 357

8 Raman C V, Pancharatnam S. The optics of mirages. Proc Indian Acad Sci A, 1959, 49: 251-261

9 Woyk E. Ray tracing theory and mirage occurrence conditions. Appl Optics, 1978, 17: 2108-2113

10 Fabri E, Fiorio G, Lazzeri F, et al. Mirage in the laboratory. Am J Phys, 1982, 50: 517-520

11 Greenler R G. Laboratory simulation of inferior and superior mirages. J Opt Soc Am A, 1987, 4: 589-590

12 Vollmer M, Tammer R. Laboratory experiments in atmospheric optics. Appl Optics, 1998, 37: 1557-1568

13 Kosa T, Palffy-Muhoray P. Mirage mirror on the wall. Am J Phys, 2000, 68: 1120-1122

14 Gutierrez D, Seron F J, Munoz A, et al. Simulation of atomospheric phenomenona. Comput Graph-UK, 2006, 30: 994-1010

15 Platt H H. Mirages? Science, 1920, 52: 290-291

16 Riggs C O. Mirage, or regular reflection? Am J Phys, 1950, 18: 526

17 Fakhruddin H. Specular reflection from a rough surface. Phys Teach, 2003, 41: 206-207

18 Tavassoly M T, Nahal A, Ebadi Z. Image formation in rough surfaces. Opt Commun, 2004, 238: 252-260

19 Zhang T. Refraction of light in media. Sci China Ser G-Phys Mech Astron, 2007, 50: 591-600

20 He Q C, Huang Y X. Reflection of plane electromagnetic waves from the surface of a perfect conductor moving in an arbitrary direction. Chinese Sci Bull, 2000, 45: 1564-1569

21 Wang X, Sun Q, Zhang X X, et al. First China ocean reflection experiment using coastal GNSS-R. Chinese Sci Bull, 2008, 53: 1117-1120

22 Rayleigh L. On the dynamical theory of gratings. Proc R Soc London Ser A, 1907, 79: 399-416

23 Beckmann P, Spizzichino A. The Scattering of Electromagnetic Waves from Rough Surfaces. New York: Pergamon Press, 1963

24 Stover J C. Optical Scattering: Measurements and Analysis. Bellingham, Washington: SPIE Press, 1995

25 Watson R M J, Raven P N. Comparison of measured BRDF data with parameterized reflectance models. Proc SPIE, 2001, 4370: 159-168

26 Fu K, Hsu P F. Modeling the radiative properties of microscale ran- 
dom roughness surfaces. J Heat Transfer, 2007, 129: 71-78

$27 \mathrm{Fu} \mathrm{K}$, Hsu P F. Radiative properties of gold surfaces with one-dimensional microscale Gaussian random roughness. Int $\mathrm{J}$ Thermophys, 2007, 28: 598-615

28 Fu K, Chen Y B, Hsu P F, et al. Device scaling effect on the spectral-directional absorptance of wafer's front side. Int J Heat Mass Transfer, 2008, 51: 4911-4925

29 Ramdas L A, Malurkar S L. Surface convection and the distribution of temperature near a heated surface. Nature, 1932, 129: 201-202

30 Tang K, Buckius R O. The geometric optics approximation for reflection from two-dimensional random rough surfaces. Int $\mathrm{J}$ Heat
Mass Transfer, 1998, 41: 2037-2047

31 Fu K, Hsu P F. New regime map of the geometric optics approximation for scattering from random rough surfaces. J Quant Spectrosc Radiat Transfer, 2008, 109: 180-188

32 Zhou H C, Sheng F, Han S D, et al. A fast algorithm for calculation of radiative energy distributions received by pinhole image-formation process from 2D rectangular enclosure. Numer Heat Transfer A, 2000, 38: 757-773

33 Lehn W H. Bright superior mirages. Appl Optics, 2003, 42: 390-393

34 Torrance K E, Sparrow E M. Theory of off-specular reflection from roughened surfaces. J Opt Soc Am, 1967, 57: 1105-1114

Open Access This article is distributed under the terms of the Creative Commons Attribution License which permits any use, distribution, and reproduction in any medium, provided the original author(s) and source are credited.

\section{Supporting information (Supplementary information)}

The supporting information is available online at csb.scichina.com and www.springerlink.com. The responsibility for scientific accuracy and content remains entirely with the authors. 